\title{
Temperature Variability and Metabolic Adaptation in Terrestrial and Aquatic Ectotherms
}

Mauricio Carter ( $\nabla$ mauricio.carter@unab.cl)

Universidad Andres Bello https://orcid.org/0000-0002-5351-8108

\section{Pablo Cortes}

Universidad Andres Bello

\section{Enrico Rezende}

Universidad Andres Bello

\section{Article}

Keywords: Global distribution, Metabolic rates, Temperature variability, Thermal adaptation, Thermodynamics

Posted Date: December 17th, 2021

DOI: https://doi.org/10.21203/rs.3.rs-1092818/v1

License: (9) This work is licensed under a Creative Commons Attribution 4.0 International License. Read Full License 


\section{Abstract}

Thermodynamics is a major factor determining rates of biochemical processes, rates of energy expenditure and ultimately resilience to global warming in ectothermic organisms. Nonetheless, whether ectothermic organisms exhibit general adaptive metabolic responses to cope with different thermal conditions remains a highly contentious subject for decades. Here we combine a model comparison approach with a global dataset of standard metabolic rates $(S M R)$, which include 1,160 measurements across 788 species of aquatic invertebrates, insects, fishes, amphibians and reptiles, to investigate the association between metabolic levels and geographic variation in environmental temperatures. According to Akaike's information criterion ( $\mathrm{AlC}_{\mathrm{c}}$ ), the variation in $S M R$ after removing allometric and thermodynamic effects is best explained by the range of temperatures encountered along the annual cycle, which provided consistently a better fit than the average temperature for the hottest and coldest month as well as mean annual temperatures. This pattern was consistent across taxonomic groups and robust to sensitivity analyses. Nonetheless, aquatic and terrestrial lineages responded differently to seasonality, with $S M R$ declining $-6.8 \%{ }^{\circ} \mathrm{C}^{-1}$ of temperature variation in aquatic organisms and increasing $2.8 \%{ }^{\circ} \mathrm{C}^{-1}$ in terrestrial. These responses reflect alternative strategies to mitigate the impact of warmer temperatures on energy expenditure, either by means of metabolic reduction in thermally stable water bodies or effective behavioral thermoregulation to exploit temperature heterogeneity on land.

\section{Introduction}

How organisms respond to their thermal environment remains a central issue in biology, spanning virtually every level of organization from rates of chemical reaction up to community- and ecosystemwide responses to global warming $(1,2)$. Thermodynamic effects on energy expenditure constitute a common theme underlying this question, and only recently have the repercussions of the exponential relationship between temperature and metabolic rates (Jensen's inequality (3)) been explored in more depth (4). Recent studies show that even a small increment in temperature, with sublethal effects from an organismic perspective, can have a major impact on energy requirements (5) and might cascade across the food chain if production and consumption rates are decoupled. Nonetheless, this is far from a new phenomenon, and different evolutionary lineages and the communities that they comprise have been continuously exposed to, and evolved under, varying thermal conditions. Thus, while the thermodynamic effects on energy expenditure rates are well understood, how temperature variation in both time and space translate into different selective pressures and how different organisms might mitigate these effects remains, to a large degree, open to debate $(6,7)$. Here, we combine the extensive information on energy expenditure available in the literature (7) with increasingly sophisticated analytical methods (6) to investigate how different descriptors of environmental thermal conditions account for the variation in metabolic rates across ectothermic organisms in a global dataset.

Following the general approach of the metabolic theory of ecology $(8,9)$, we can express the effects of body mass $M$ and measurement temperature $T_{a}$ (= body temperature in ectothermic organisms) in 
standard metabolic rates $S M R$ as

$S M R=a M^{b} 10^{I_{a} \log Q_{10}}$ :io

(eqn 1)

where $Q_{10}$ corresponds to the thermal sensitivity of biological rates to a change in $T_{a}$ of $10{ }^{\circ} \mathrm{C}$ (or $10 \mathrm{~K}$ ), which is usually around two or three for physiological systems (we work with $\log _{10}$ to express variation in $S M R$ and $M$ in orders of magnitude). This general relationship can be expressed in linear form employing logarithms, and the effects of $M$ and $T_{a}$ across lineages partitioned with a linear model of the form

$\log S M R=\log a+b \log M+T_{a} / 10 \log Q_{10}+\varepsilon$

where $\varepsilon$ corresponds to the residual error, which may vary predictably with other environmental variables under the premise that this variation is adaptive.

Many studies in the literature have employed variants of this model to test different adaptive scenarios, such as the metabolic cold adaptation hypothesis, which posits that species from cold environments have evolved higher SMR to compensate for thermodynamic restraints, that has received considerable attention in the physiological ecology literature $(10,11)$. Results from different studies are far from conclusive, and are often confounded by a variety of factors such as taxonomic affiliation, level of organization, range of environmental temperatures encountered and methodological approach. To determine which variables might account for variation in metabolic rates on a global scale, we compiled data on body mass, SMR and measurement $T_{a}$ from previous reviews on aquatic invertebrates, insects, fishes, amphibians and reptiles $(10,12-14)$, and obtained four environmental thermal proxies by mapping their sampling locations onto data grids with air and sea surface temperatures (see Methods): the average temperature of the coldest month $T_{\min }$, of the warmest month $T_{\text {max }}$, their average $T_{\text {mean }}$ and their range $T_{\text {range }}$ (Figs. S1 and S2). The resulting dataset contained 1,160 measurements across 788 species spanning a latitudinal gradient from $76^{\circ} 22^{\prime} \mathrm{S}$ to $78^{\circ} 49^{\prime} \mathrm{N}$ (Fig. 1a). Importantly, removing marine organisms inhabiting depths below $50 \mathrm{~m}$, that might be subject to oxygen limitation (15) or whose thermal environment may deviate substantially from sea surface temperatures, do not change the outcome of analyses hence we report results for the full dataset.

Regressions including only allometric and $T_{a}$ effects (Fig. 1b,c) give rise to $b=0.77 \pm 0.01$ ( \pm SE) and back-transformed $Q_{10}=2.71 \pm 0.01$, whereas controlling for taxonomic group as a fixed factor we obtain $b=0.79 \pm 0.01$ and $Q_{10}=2.58 \pm 0.01$, which fall in the typical range reported in the literature. Visual inspections indicate that log $S M R$ varies linearly with $T_{a}$ (Fig. 1c) and that the model comparison approach employing general linear models is statistically sound (i.e., while we expect log SMR to eventually drop due to heat stress, this effect was not evident here). Based on Akaike's information criterion $\left(\mathrm{AlC}_{\mathrm{C}}\right)$, regression models that included body mass and $T_{\text {range }}$ provided the best fit to $S M R$, and this was true for the pooled dataset and for analyses performed separately for each of the major groups mentioned above (Table S1 and S3). Interestingly, while $T_{\text {range }}$ was consistently a better explanatory 
variable than $T_{\text {mean }}, T_{\text {max }}$ and $T_{\text {min }}$, the relationship between residual $S M R$ and $T_{\text {range }}$ was positive in terrestrial insects, amphibians and reptiles and negative in aquatic invertebrates and fish (Fig. 1d-h). Within aquatic invertebrates, this negative trend is also observed in separate analyses for copepods, decapods and amphipods (Fig. S3). Accordingly, the interaction between $T_{\text {range }}$ and a binary factor coding for terrestrial $v s$ aquatic exhibited the largest effect size apart from body mass and $T_{a}$ (Table S2 and S4), which is not entirely surprising given that these organisms encounter very different levels of thermal variability and $\mathrm{O}_{2}$ demand-supply constraints $(16,17)$. Separate regressions for terrestrial and aquatic organisms supported $T_{\text {range }}$ as the best predictor of $S M R$ in both cases, with significant positive and negative slopes, respectively. In other words, $S M R$ tends to increase with $T_{\text {range }}$ in terrestrial and decrease in aquatic organisms (Fig. 2a,b). Sensitivity analyses employing 10,000 random subsets with $50 \%$ of the dataset each show that this outcome is highly robust to sampling effects and variation in $Q_{10}$ (Fig. 2c,d) largely reducing the possibility that results are caused by influential points, phylogenetic overrepresentation, or biases of other sorts, and $T_{\text {range }}$ resulted consistently in the best models based on their lower AIC (see Methods). Based on slope averages from these 10,000 replicates, for a terrestrial organism measured at $T_{a}=25^{\circ} \mathrm{C}$, mass-independent $S M R$ increases at a rate of $2.8 \%^{\circ} \mathrm{C}^{-1}$ of seasonal variation, whereas for its aquatic counterpart it decreases as $-6.8 \%^{\circ} \mathrm{C}^{-1}$. In relative terms, this implies that a change in $T_{\text {range }}$ from 0 to $20^{\circ} \mathrm{C}$ is associated with an average increase in SMR from 1.00 to 1.74 in terrestrial organisms and a drop to $~ 0.24$ in aquatic ones, although this magnitude varies with body size and across taxa as evidenced by significant interaction effects (Tables S1 and S3). Results remain similar if amphibians are classified as aquatic instead of terrestrial.

Previous analyses indicate that $S M R$ increases with latitude (18), which could partly reflect variation in environmental temperatures in general and $T_{\text {range }}$ in particular (Fig. S1). The contrasting relationships between residual $S M R$ and $T_{\text {range }}$ in terrestrial versus aquatic organisms, and this variable being consistently selected as a better predictor than other temperature indices (Tables S1 - S4), point towards the existence of different strategies to cope with thermal variation. Interestingly, terrestrial ectotherms from highly seasonal environments tend to increase their $S M R$, while aquatic organisms exhibit the opposite strategy and reduce their SMR (Fig. 2a,b). We argue that this contrast partly emerges as a response to different levels of temperature heterogeneity in these environments (18) and the possibility to thermoregulate by behavioral means, as illustrated by analyses employing air and water temperature records for Bassenthwaite Lake, U.K., in 2010 (19). In this lake air temperatures fluctuate substantially more than water temperatures on a daily basis, with temperatures dropping at night (Fig. 3a). In such a variable thermal environment, evolving higher $S M R$ impinges on relatively low costs in average daily energy expenditure (a 39\% increase from $0.76 \times 10^{-4}$ to $1.06 \times 10^{-4} \mathrm{~W}$ based on adjusted SMR estimates), favoring behavioral thermoregulation and a 'on/off metabolic strategy that maximizes performance when it is warm and shuts down expenditure in the cold. Accordingly, our sensitivity analysis for terrestrial lineages shows that $T_{\min }$ provides a better fit to residual $S M R$ than $T_{\text {max }}$ with a negative slope that implies that $S M R$ increases in regions with colder extremes (Fig. 2c). In contrast, the negative relationship between $S M R$ and $T_{\text {range }}$ in aquatic environments may reflect selection to reduce aerobic 
metabolism during the warm season, which can result in energy saving of $80.8 \%$ (from $3.0 \times 10^{-4}$ to 0.57 $\times 10^{-4} \mathrm{~W}$ ) (Fig. 3e). Indeed, in aquatic organisms $T_{\max }$ provided a better fit to $S M R$ than $T_{\min }$, with a negative relationship that supports this interpretation (Fig. 2d).

Which factors might explain the observed trends? Terrestrial and aquatic thermal environments exhibit qualitatively different patterns of daily and seasonal variation (Figs. 3 and S4), which explains why there are thriving aquatic communities and only a handful of terrestrial ectothermic lineages near the poles. On land, subfreezing temperatures in seasonal environments restricts the growing period, hence lineages in these regions should evolve higher metabolic rates to maximize biomass production (a.k.a., energetic fitness) in the narrower window when temperatures are warm, via higher fecundity, growth rates and/or reduced generation times (20). The 'on/off strategy may be evolutionarily advantageous in terrestrial seasonal environments because animals can maximize aerobic performance at low energy costs, though this strategy should be constrained for larger terrestrial ectotherms due to thermal inertia. This prediction is supported both within taxonomic groups, based on the significant and negative interaction between $M$ and $T_{\text {range }}$ in separate linear models for insects and reptiles, and across groups with the relationship between $S M R$ and $T_{\text {range }}$ being more pronounced in insects than reptiles (Fig. 1). Interestingly, larger ectotherms from bumblebees to alligators bask in the sun to warm up, which is an 'on/off strategy that is possible primarily on land, which supports our contention that terrestrial ectotherms take advantage of the inherent thermal variability of terrestrial environments to maximize their fitness. Crucially, we assume that $S M R$ is an adequate proxy of biomass production after removing the effects of varying activity levels, foraging strategies or mode of locomotion. Nonetheless, how variation in SMR correlates with lifehistory traits such as growth, fecundity and survival is still a matter of debate (20), even more so because this relationship may change with food availability (21).

In aquatic environments the situation is more complex because $T_{\text {range }}$ is low in both tropical and polar regions (Figs. S1 and S2), hence different factors likely explain the elevated SMR of organisms from these contrasting environments. That polar lineages exhibit higher $S M R$ is coherent with patterns for terrestrial organisms and the metabolic cold adaptation hypothesis (22), assuming that elevated SMR reflects higher biomass production. However, this group includes only a few data $(n=23)$. Instead, the stronger support to models with $T_{\max }$ over $T_{\min }$ and its negative association with $S M R$ (Fig. 2d,f) suggests that high temperatures constrain elevated $S M R$ in several lineages, possibly mediated by $\mathrm{O}_{2}$ availability. Large-scale analyses indicate that reductions in size associated with warming temperatures are more pronounced in aquatic then terrestrial organisms $(16,17)$, presumably due to limitations in $\mathrm{O}_{2}$ supply in aquatic environments $(23,24)$, hence a reduced mass-independent $S M R$ may constitute a complementary response to this problem. Lower $S M R$ were observed in aquatic invertebrates and fishes at intermediate latitudes where $T_{\text {range }}$ is maximal (between $20^{\circ}$ and $50^{\circ}$, Fig. S1), which ultimately explains why models with $T_{\text {range }}$ provide a better fit than those with $T_{\text {max }}$ from a statistical perspective. Mechanistically, selection may be stronger in seasonal environments because organisms must adjust not only to temperatures that can vary up to $20^{\circ} \mathrm{C}$ between summer and winter, but also to variation in food availability, seasonal or episodic hypoxia (13) and mismatch between these variables. Ultimately, our 
results appear to be complementary to previous studies reporting contrasting latitudinal trends in body size distribution and responses to warming conditions across terrestrial and aquatic organisms $(16,17)$, and suggest that thermal variability (or the absence thereof) plays an important role shaping $S M R$ in both aquatic and terrestrial ectotherms.

To explore the generality of these findings, we partitioned the dataset into smaller subsets and tested a set of related hypotheses stemming from our proposed conceptual framework (Fig. S4). Specifically, we assessed if the association between $T_{\text {range }}$ and $S M R$ varies significantly (1) with $T_{\text {mean }}$ in both aquatic and terrestrial organisms because the selective pressures proposed above should vary with both the temperature means and extremes, (2) with spatial variation in sun/shade, estimated with the normalized difference vegetation index (NDVI) for both terrestrial and freshwater organisms (i.e., aquatic organisms in continental waters), since habitats with different vegetation cover should exhibit contrasting levels of thermal variation, (3) with daily thermal range ( $\left.D T_{\text {range }}\right)$ in terrestrial but not aquatic organisms (Fig. 3), and (4) between aquatic organisms in freshwater versus marine systems for essentially the same reason. Employing a model comparison approach and testing these predictions in separate subsets of organisms we were able to perform a total of seven independent tests (Table 1). In all cases, the inclusion of one additional variable in the standard model controlling for $T_{\text {range }}$ effects resulted in a substantial increase in model fitness, as indicated by their lower $\mathrm{AlC}_{\mathrm{c}}$ and Akaike weights $w_{i}>0.98$ (which indicates that these models have a support of over $98 \%$ when compared against the simpler model including only $T_{\text {range) }}$. While these models are difficult to interpret in more detail because they contain many interactions, and the adaptive significance of alternative metabolic strategies will vary with the natural histories of the animals, their habitat characteristics and other factors that are not encapsulated in our coarse variables, these analyses clearly suggest that temperature variability is a major factor driving metabolic evolution in geographic scales.

To summarize, analyses described here show that rates of energy expenditure across ectothermic organisms are explained to large degree by the variability in thermal conditions that they encounter on a daily and seasonal basis, and contrasting responses between terrestrial and aquatic organisms. Results are robust when models are run separately for different taxonomic groups and according to sensitivity analyses, and suggest genuine adaptive variation to differences in temperature variability (25). If so, climate forecasts might be employed to study which metabolic strategies might be favored in aquatic and terrestrial environments across different regions of the globe. In our opinion, the general responses reported here contrast with the fragmented, and often inconclusive, evidence provided to support alternative hypotheses of metabolic temperature adaptation (26-29) and puts this subject, from a mechanistic perspective, to rest on more solid ground. Importantly, while multiple tests provide some support to our conjecture that temperature variability is an important factor driving metabolic evolution, our proposed framework for the observed differences between terrestrial and aquatic organisms does not exclude alternative scenarios or synergistic effects (which is particularly true for aquatic organisms, where $\mathrm{O}_{2}$ availability may impose metabolic constraints that might ultimately underlie the general findings reported here). In retrospect, this is not entirely surprising. Organisms must survive and 
reproduce within a confined thermal window and, therefore, it is reasonable to expect that the variation in metabolic levels across different lineages reflect concerted responses to cope with both upper and lower thermal extremes that they encounter in their respective environments.

\section{Methods}

We compiled data on $S M R$ from previous reviews on aquatic invertebrates (12), insects (10), fishes (13), amphibians (30) and reptiles (14). All datasets provided body mass, $S M R$ and $T_{a}$, with the exception of insects whose $S M R$ was already adjusted to $T_{a}=25^{\circ} \mathrm{C}$ assuming a $Q_{10}=2.0$ (10). While this prevented a more complete analysis of $T_{a}$ effects in insect $S M R$, we could still test for adaptive variation in $S M R$ to temperature differences across environments, which constituted the main purposes of this study. All SMR were transformed to Watts employing a conversion factor of $1 \mathrm{ml} \mathrm{O}_{2}=20.083 \mathrm{~J} \mathrm{(31).}$

From the original references in these reviews, we obtained the geographic location where the source populations were collected and, with this information, estimated the mean maximum and minimum mean monthly temperatures experienced by each population/species from data grids with air (32) and sea surface temperatures (33) available at the NOAA webpage (https://www.esrl.noaa.gov/psd/data/gridded/) with a $0.5^{\circ} \times 0.5^{\circ}$ resolution (Figs. 1 , S1 and S2). We only included those lineages whose geographic origin could be readily established and that were maintained at most only a few generations in the laboratory, and employed sea surface temperatures to characterize the thermal environment of marine organisms (i.e., several fish and aquatic invertebrates) and air temperatures for everything else (i.e., terrestrial organisms or those inhabiting continental rivers and lakes). While these coarse variables may not perfectly reflect the range of microclimates available in different terrestrial or aquatic habitats, the use of monthly means for the warmest $\left(T_{\max }\right)$ and coldest month $\left(T_{\min }\right)$ does provide a good estimation of the range of temperatures that these ectothermic organisms should encounter in the field over the yearly cycle (Fig. 3). To avoid potentially influential points from abnormally cold regions, we set temperatures below zero $=0^{\circ} \mathrm{C}$ and therefore assumed that at these temperatures, metabolism in no longer under selection because animals are either frozen or in diapause. While this affected a few marine polar species, the difference in temperature estimates were < $1^{\circ} \mathrm{C}$ and had no impact in the analyses. With this approach, we estimated $T_{\max }$ and $T_{\text {min }}$, and also $T_{\text {mean }}$ and $T_{\text {range }}$ as, respectively, the average or the difference between these two indices, for a total of 1,160 measurements ( $n=788 \mathrm{spp}$ ) from the 1,896 originally listed in the different reviews.

To account for potential confounding effects of water depth and vertical distribution of marine organisms in our analyses, we obtained information on minimum and maximum depth for 204 of the 279 marine species in our dataset from the original sources or online (www.sealifebase.se), and repeated all analyses with a conservative dataset including only those species encountered near the surface (i.e., minimum depths $<50 \mathrm{~m}, n=133 \mathrm{spp}$ ). This procedure did not change qualitatively the outcome of analyses and the general conclusions, hence for simplicity we report results for the whole dataset. 


\section{Statistical analyses}

We employed a model comparison approach (34) to select the best model that explains the variation in $S M R$. Analyses were performed for both the entire dataset and separately for each of the groups studied in the different reviews (aquatic invertebrates, insects, fishes, amphibians and reptiles). We used Akaike Information Criterion for small samples ( AIC $_{\mathrm{c}}$ ) to select the best model, and a hierarchical approach to test incrementally more complex models, from a purely allometric model up to different models with $Q_{10}$ varying across lineages and testing for effects of the thermal environment in the following form:

$\log S M R \sim \log M+T_{a}+$ Taxa $+T_{\text {field }}($ eqn 3)

where $M$ corresponds to the body mass (g), $T_{a}$ to measurement temperature $\left({ }^{\circ} \mathrm{C}\right)$, Taxa to a factor identifying the taxonomic group studied and $T_{\text {field }}$ to one of the four descriptors of thermal environment obtained for each organism ( $T_{\text {mean }}, T_{\min }, T_{\max }$ and $T_{\text {range }}$ expressed in $\left.{ }^{\circ} \mathrm{C}\right)$. To obtain the models with increasing complexity, the independent variables and factors were included incrementally from left to right, concomitantly with all pairwise interactions (higher order interactions were excluded to ease interpretation). The relative support of each model was obtained with Akaike weights $\left(w_{i}\right)$, which quantifies the probability that each particular model is the correct one when compared against all models being tested. With this stepwise approach, we assessed how each factor, namely body size, a constant versus a variable $Q_{10}$, taxonomic group, and the four different descriptors of thermal environment, contributes to $S M R$. For the model with the lowest AIC $_{\mathrm{c}}$ (i.e., the model with the best fit), we employed Cohen's $f$ to quantify the effect size and relative contribution of main effects and pairwise interactions to the overall variation in SMR. To determine to what extent differences in SMR across taxonomic groups could be explained by habitat type, we repeated these analyses including, instead of Taxa, separate aquatic $v s$ terrestrial and vertebrate $v s$ invertebrate binary factors. Additionally, we repeated these analyses employing mixed models, using species identity as random effect to consider repeated measurements for a single species (which was not an issue for fishes and reptiles). Because results remain qualitatively identical, here we discuss the results of the GLM models for clarity and list the mixed model results in the supplementary material. We employed GLM adjusted estimates of SMR, in combination with air and water temperatures for Bassenthwaite Lake, U.K. (10 m depth) (19), to illustrate how differences in daily temperature variation impact energy expenditure in terrestrial and aquatic environments.

We then performed a sensitivity analysis to determine how robust results are to sampling effects, accounting for potential biases in phylogenetic representation, geographic origins and also possible differences in $Q_{10}$ across species (with the caveat that in the insect dataset this was not possible). With this goal, we obtained 10,000 random subsets comprising $50 \%$ of the original $S M R$ dataset $(n=580$ for each subset) and calculated $S M R$ standardized to $25^{\circ} \mathrm{C}$ by assigning a random $Q_{10}$ from a uniform distribution ranging between 1.5 and 3.5. Importantly, here we allow $Q_{10}$ to vary across every single measure, relaxing the assumption that $Q_{10}$ remains constant within grouping factors defined a priori (i.e., 
across Taxa or aquatic vs terrestrial). Subsequently, we compared the slope and $\mathrm{AlC}_{\mathrm{c}}$ estimates from the four full general linear models described above including $T_{\text {mean }}, T_{\text {min }}, T_{\text {max }}$ and $T_{\text {range }}$ as the environmental descriptor (in this analysis we focused on main effects and pairwise interactions were removed for simplicity). To obtain a distribution of $\mathrm{AlC}_{\mathrm{c}}$ across replicates in a comparable scale, we centered the average $\mathrm{AlC}_{\mathrm{c}}$ obtained in each replicate to zero and express results for each model as the $\triangle \mathrm{AIC}_{\mathrm{c}}$ from this mean. With this approach, we estimated the variation in effect size (slope) for each of the environmental descriptors and determined if results from the model comparison approach were consistent across smaller and different datasets.

Finally, we tested if the inclusion of an additional predictor would improve the model with the best fit obtained with the methodology above, based on the different hypotheses proposed for terrestrial and aquatic organisms (see above). With this purpose, we separated the dataset into terrestrial and aquatic organisms and compared a basal model including only $T_{\text {range }}$ against the expanded model:

$\log S M R \sim \log M+T_{a}+$ Taxa $+T_{\text {range }}+$ Env (eqn 4)

where Env corresponds to an additional environmental predictor that varies with the specified hypothesis. Specifically, we included the continuous variables $T_{\text {mean, }} N D V I$, daily thermal range $D T_{\text {range, }}$ a factor coding for freshwater versus marine environments (Table 1). Annual means for NDVI with a $1^{\circ} \times 1^{\circ}$ resolution and daily thermal ranges with a $0.5^{\circ} \times 0.5^{\circ}$ resolution were obtained from data grids described in http://research.jisao.washington.edu/data/ndvi/ and (35), respectively. As described above (eqn 3), comparisons between models with and without Env were performed comparing $\mathrm{AlC}_{\mathrm{c}}$ and only pairwise interactions were allowed. We concluded that the proposed hypotheses are supported if their respective models including Env provided a better fit (i.e., a lower $\mathrm{AlC}_{\mathrm{c}}$ ) than the basal model, and ignored the sign of the coefficient as means to test for directionality given the high number of significant interactions in these models. All analyses were performed in R (http://cran.r-project.org) and results for the different models are provided in detail in the Supplementary Online Material (Tables S1 - S5). 
Table 1

Testing different adaptive hypotheses proposed to explain the variation in SMR across terrestrial and aquatic ectotherms.

\begin{tabular}{|c|c|c|c|c|}
\hline \multirow[t]{2}{*}{ Subset } & Additional & $\Delta \mathrm{AIC}_{\mathrm{c}} \S$ & $w_{i} \S$ & Prediction \\
\hline & \multicolumn{4}{|l|}{ Variable } \\
\hline \multirow[t]{3}{*}{ Terrestrial $(n=616)$} & $T_{\text {mean }}$ & -9.76 & 0.99 & $\sqrt{ }$ \\
\hline & $N D V I$ & -7.93 & 0.98 & $\sqrt{ }$ \\
\hline & $D T_{\text {range }}$ & -11.1 & 1.00 & $\sqrt{ }$ \\
\hline \multirow[t]{2}{*}{ Aquatic $(n=544)$} & $T_{\text {mean }}$ & -58.01 & 1.00 & $\sqrt{ }$ \\
\hline & Fresh vs marine & -7.41 & 0.98 & $\sqrt{ }$ \\
\hline \multirow[t]{2}{*}{ Freshwater $(n=48)$} & $N D V I$ & -11.04 & 1.00 & $\sqrt{ }$ \\
\hline & $D T_{\text {range }} \S \S$ & +13.38 & 0.00 & $\sqrt{ }$ \\
\hline \multicolumn{5}{|c|}{$\S$ All models compared against $\log S M R \sim \log M+T_{a}+$ Taxa $+T_{\text {range }}$ (see Methods). } \\
\hline \multicolumn{5}{|c|}{$\begin{array}{l}\S \S \text { Note that } D T_{\text {range }} \text { is expected not to improve the model here, in opposition to terrestrial organisms } \\
\left.\text { where } D T_{\text {range }} \text { should explain some variation in } S M R \text { (Fig. } 3\right) \text {. }\end{array}$} \\
\hline
\end{tabular}

\section{Declarations}

\section{Additional information}

Supplementary information is available in the online version of the paper. Reprints and permissions information is available online at www.nature.com/reprints. Correspondence and requests for materials should be addressed to G.W.

\section{Competing financial interests}

The authors declare no competing financial interests.

\section{References}

1. J. C. Quijano, H. Lin, Is spatially integrated entropy production useful to predict the dynamics of ecosystems? Ecol. Modell. 313, 341-354 (2015).

2. G. S. F. da Silva, M. L. Glass, L. G. S. Branco, Temperature and respiratory function in ectothermic vertebrates. J. Therm. Biol. 38, 55-63 (2013).

3. J. G. Liao, A. Berg, Sharpening Jensen's Inequality. Am. Stat. 73, 278-281 (2019). 
4. V. M. Savage, Improved approximations to scaling relationships for species, populations, and ecosystems across latitudinal and elevational gradients. J. Theor. Biol. 227, 525-534 (2004).

5. M. E. Dillon, G. Wang, R. B. Huey, Global metabolic impacts of recent climate warming. Nature 467, 704-706 (2010).

6. P. M. Schulte, T. M. Healy, N. A. Fangue, Thermal performance curves, phenotypic plasticity, and the time scales of temperature exposure. Integr. Comp. Biol. 51, 691-702 (2011).

7. W. W. Dowd, F. A. King, M. W. Denny, Thermal variation, thermal extremes and the physiological performance of individuals. J. Exp. Biol. 218, 1956-1967 (2015).

8. J. F. Gillooly, J. H. Brown, G. B. West, V. M. Savage, E. L. Charnov, Effects of Size and Temperature on Metabolic Rate. Nature 293, 1-6 (2001).

9. J. H. Brown, J. F. Gillooly, A. P. Allen, V. M. Savage, G. B. West, Toward a metabolic theory of ecology. Ecology 85, 1771-1789 (2004).

10. S. L. Chown, et al., Scaling of insect metabolic rate is inconsistent with the nutrient supply network model. Funct. Ecol. 21, 282-290 (2007).

11. C. R. White, P. B. Frappell, S. L. Chown, An information-theoretic approach to evaluating the size and temperature dependence of metabolic rate. Proc. R. Soc. B Biol. Sci. 279, 3616-3621 (2012).

12. A. M. Makarieva, et al., Mean mass-specific metabolic rates are strikingly similar across life's major domains: Evidence for life's metabolic optimum. Proc. Natl. Acad. Sci. U. S. A. 105, 16994-16999 (2008).

13. S. S. Killen, et al., Ecological Influences and Morphological Correlates of Resting and Maximal Metabolic Rates across Teleost Fish Species. Am. Nat. 187, 592-606 (2016).

14. C. R. White, N. F. Phillips, R. S. Seymour, The scaling and temperature dependence of vertebrate metabolism. Biol. Lett. 2, 125-127 (2006).

15. J. W. F. Chu, K. S. P. Gale, Ecophysiological limits to aerobic metabolism in hypoxia determine epibenthic distributions and energy sequestration in the northeast Pacific ocean. Limnol. Oceanogr. 62, 59-74 (2017).

16. J. Forster, A. G. Hirst, D. Atkinson, Warming-induced reductions in body size are greater in aquatic than terrestrial species. Proc. Natl. Acad. Sci. U. S. A. 109, 19310-19314 (2012).

17. C. R. Horne, A. G. Hirst, D. Atkinson, Temperature-size responses match latitudinal-size clines in arthropods, revealing critical differences between aquatic and terrestrial species. Ecol. Lett. 18, 327335 (2015).

18. J. P. DeLong, et al., Habitat, latitude and body mass influence the temperature dependence of metabolic rate. Biol. Lett. 14 (2018).

19. I. D. Jones, H. Feuchtmayr, S. C. Maberly, Data from automatic water monitoring buoy from Bassenthwaite Lake, 2012 to 2015. NERC Environmental Information Data Centre (2017).

20. J. Robert Burger, C. Hou, C. A. S. Hall, J. H. Brown, Universal rules of life: metabolic rates, biological times and the equal fitness paradigm. Ecol. Lett. 24, 1262-1281 (2021). 
21. S. K. Auer, et al., Metabolic rate interacts with resource availability to determine individual variation in microhabitat use in the wild. Am. Nat. 196, 132-144 (2020).

22. C. R. White, L. A. Alton, P. B. Frappell, Metabolic cold adaptation in fishes occurs at the level of whole animal, mitochondria and enzyme. Proc. R. Soc. B Biol. Sci. 279, 1740-1747 (2012).

23. D. Pauly, W. W. L. Cheung, Sound physiological knowledge and principles in modeling shrinking of fishes under climate change. Glob. Chang. Biol. 24, e15-e26 (2018).

24. J. G. Rubalcaba, W. C. E. P. Verberk, A. Jan Hendriks, B. Saris, H. Arthur Woods, Oxygen limitation may affect the temperature and size dependence of metabolism in aquatic ectotherms. Proc. Natl. Acad. Sci. U. S. A. 117, 31963-31968 (2020).

25. G. Wang, M. E. Dillon, Recent geographic convergence in diurnal and annual temperature cycling flattens global thermal profiles. Nat. Clim. Chang. 4, 988-992 (2014).

26. F. Bozinovic, P. Calosi, J. I. Spicer, Physiological Correlates of Geographic Range in Animals. Annu. Rev. Ecol. Evol. Syst. 42, 155-179 (2011).

27. E. L. Rezende, F. Bozinovic, T. G. Jr, Climatic Adaptation and the Evolution of Basal and Maximum Rates of Metabolism in Rodents. Evolution (N. Y). 58, 1361-1374 (2004).

28. A. Addo-Bediako, S. L. Chown, K. J. Gaston, Metabolic cold aadaptation: a large-scale perspective. Funct. Ecol. 16, 332-338 (2002).

29. S. L. Chown, K. J. Gaston, Macrophysiology - progress and prospects. Funct. Ecol. 30, 330-344 (2016).

30. R. E. Gatten, K. J. Miller, R. J. Full, "Energetics at Rest and during Locomotion” in Environmental Physiology of the Amphibians, M. E. Feder, W. W. Burggren, Eds. (University of Chicago Press, 1992), pp. 314-374.

31. K. Schmidt-Nielsen, Animal Physiology: Adaptation and Environment (Cambridge University Press, 1997).

32. Y. Fan, H. van den Dool, A global monthly land surface air temperature analysis for 1948-present. J. Geophys. Res. Atmos. 113, 1-18 (2008).

33. R. W. Reynolds, N. A. Rayner, T. M. Smith, D. C. Stokes, W. Wang, An improved in sotu and satellite SST analysis for climate. J. Clim. 15, 1609-1625 (2002).

34. K. P. Burnham, D. R. Anderson, Model selection and multimodel inference: a practical informationthoeretic approach (Springer, 2002).

35. I. Harris, P. D. Jones, T. J. Osborn, D. H. Lister, Updated high-resolution grids of monthly climatic observations - the CRU TS3.10 Dataset. Int. J. Climatol. 34, 623-642 (2014).

\section{Figures}



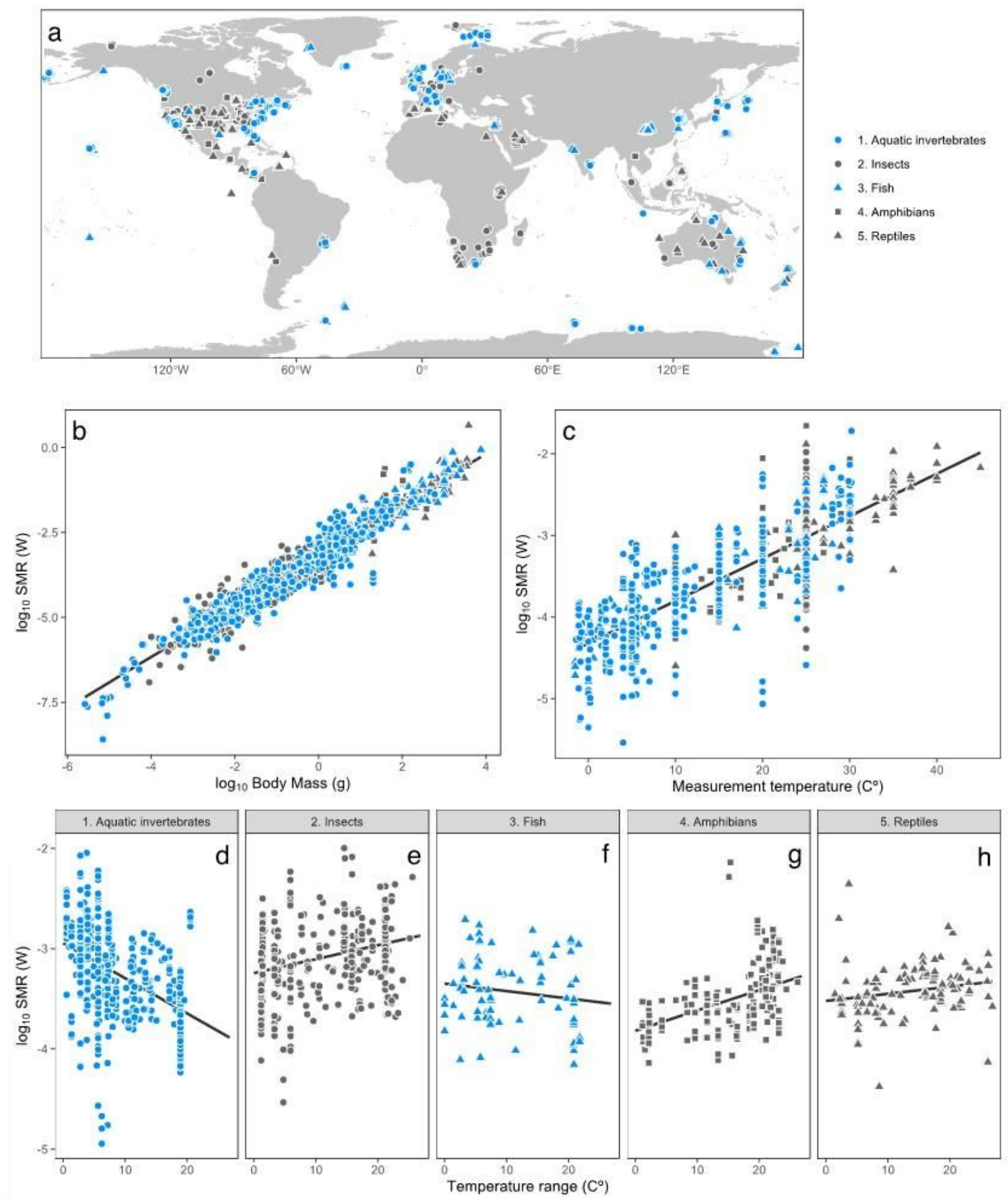

\section{Figure 1}

Collection localities and SMR variation in ectotherms. Sample locations (a). The best model explaining the variation in SMR include allometric (b) and thermodynamic effects (c), taxonomic affiliation and its interaction with the seasonal variation in temperature $T_{\text {range }}$ (see Methods). Partial plots illustrate these effects controlling for the remaining variables in the model, hence values are calculated after readjusting these variables to their means $(\mathbf{d}-\mathbf{h})$. 

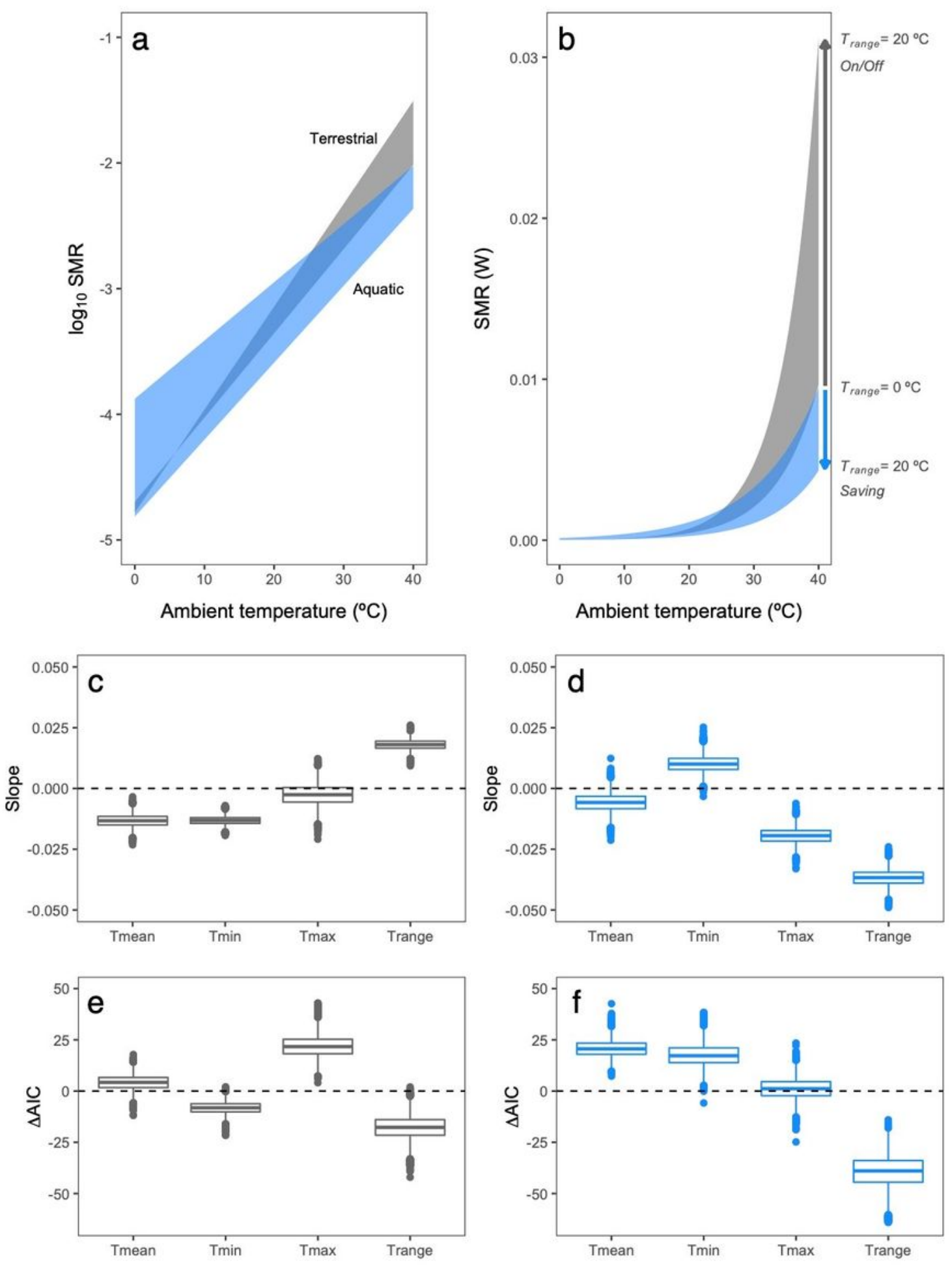

\section{Figure 2}

Metabolic responses in terrestrial versus aquatic environments and sensitivity analyses. Thermal sensitivity in $S M R$ calculated from adjusted estimates assuming a body mass of $0.44 \mathrm{~g}$ and $T_{\text {range }}$ varying between 0 and $20^{\circ} \mathrm{C}$, expressed in log-scale (a) and linear scale (b). The shaded areas encapsulate the differences in response between terrestrial and aquatic organisms adapted to these contrasting values of $T_{\text {range. }}$. This contrasting response between terrestrial and aquatic ectotherms to 
seasonal variation in temperature is detected in sensitivity analyses, replicating 10,000 times the linear models with random subsets of the dataset. The partial regression slope between $S M R$ and $T_{\text {range }}$ was consistently positive for terrestrial (c) and negative for aquatic organisms (d), and models with $T_{\text {range }}$ consistently result in the lowest $\mathrm{AIC}_{\mathrm{C}}$ for terrestrial $(\mathbf{e})$ and aquatic lifestyles $(\mathrm{f}) . \triangle \mathrm{AIC}_{\mathrm{C}}$ are calculated against the overall $\mathrm{AIC}_{\mathrm{C}}$ mean of each comparison to make results comparable across replicates (see Methods).
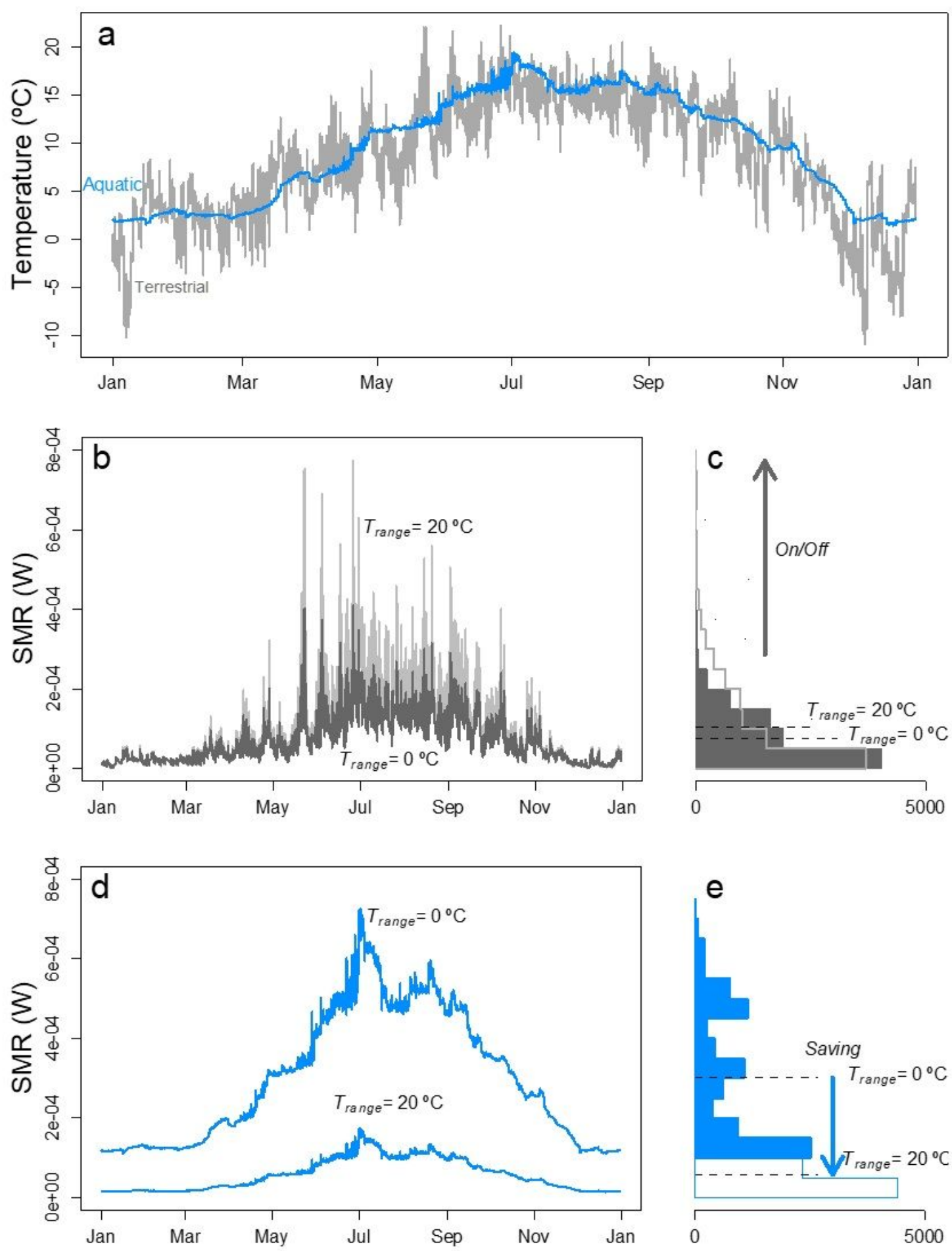


\section{Figure 3}

Metabolic responses to seasonal temperature variation. Air and water temperature profile from Bassenthwaite Lake, U.K., recorded with a 1-h resolution in 2010 (a), employed to calculate how SMR varies with temperature in terrestrial (b) and aquatic environments (d). Note how adjusted estimates from our model (Figs. 2a,b) translate into contrasting behaviors between these environments: terrestrial organisms from seasonal environments $\left(T_{\text {range }}=20^{\circ} \mathrm{C}\right)$ employ an 'on/off strategy that allows them to increase $S M R$ and exploit periods of warm temperatures at low energy costs (b, c), whereas aquatic organisms exhibit lower $S M R$ that results in substantial energy savings during warm conditions (d, e). The dotted lines represent mean energy expenditure for the different groups.

\section{Supplementary Files}

This is a list of supplementary files associated with this preprint. Click to download.

- ThermaladaptationSINN.docx 